Background and aims Device-related infections are thought to be initiated by adhesion of the bacteria to a medical device, followed by colonisation and mature biofilm formation. Preterm infants are susceptible to device-related infections caused by Staphylococcus epidermidis. Also, preterm infants have lower levels of antimicrobial peptides, including human cathelicidin antimicrobial peptide LL37, a condition that in part may explain their increased vulnerability. Our aim was to evaluate the effect of peptide LL37 on 1) the expression of biofilm-associated genes and 2) biofilm mass, by using an in vitro model.

Methods Biofilm formation of $S$. epidemidis was studied on intra vascular catheter pieces and in culture plates, in the absence or presence of LL37. Bacterial biofilm mass was investigated by scanning electron microscopy (SEM). Changes in biofilm-associated gene expression was determined by real-time polymerase chain reaction.

Results Tissue-like concentration of the peptide down-regulated most of the investigated genes after $2 \mathrm{~h}$. A diminished biofilm mass was seen on the catheter surface by SEM after $24 \mathrm{~h}$ incubation.

Conclusions Peptide LL37, as part of innate immune defense of the newborn infant is crucial for the regulation of the commensal flora, including Staphylococcus epidermidis. A diminished activity of LL37, as found in preterm infants, may contribute to increase their risk of device-related infections.

\section{PS-303 VITAMIN D INCREASES ROI PRODUCTION IN TERM AND PRETERM INFANTS: POSSIBLE MECHANISM OF ENHANCED ANTIBACTERIAL EFFECT}

${ }^{1} \mathrm{C}$ Onwuneme, ${ }^{1} \mathrm{~F}$ Martin, ${ }^{2} \mathrm{~A}$ Blanco, ${ }^{2} \mathrm{~A}$ O'Neill, ${ }^{3} \mathrm{~N}$ Murphy, ${ }^{2} \mathrm{RW}$ W Watson, ${ }^{1} \mathrm{EJ}$ Molloy. ${ }^{1}$ Neonatology, National Maternity Hospital, Dublin, Ireland; ${ }^{2}$ School of Medicine and Medical Sciences, Conway Institute University College Dublin, Dublin, Ireland; ${ }^{3}$ Paediatrics Endocrinology, Children's University Hospital Temple Street, Dublin, Ireland

\subsection{6/archdischild-2014-307384.602}

Introduction Newborn infants are at risk of vitamin D deficiency and various studies implicate vitamin D as having immunomodulatory effect. Adequate generation of reactive oxygen intermediates (ROI) by neutrophils (PMN) during sepsis is bactericidal. However, production of neutrophil oxidase activity in the presence of sepsis is impaired in neonates.

Aim To examine the in vitro effect of $1,25(\mathrm{OH})_{2} \mathrm{D}_{3}$ on whole blood PMN and monocyte ROI, TLR4, CD11b in newborn infants during sepsis in vitro.

Methods Whole blood from preterm infants $<32$ weeksgestation within $24 \mathrm{~h}$ of birth, cord blood from term infants and adult controls were analysed for phagocytic expression of Toll-Like Receptor 4 (TLR4; pathogen recognition); CD11b (chemotaxis and adhesion) and ROI production (bacterial kill) using flow cytometry. These were assessed in response to Lipopolysaccharide (LPS; Endotoxin; in vitro sepsis) and 1,25(OH) ${ }_{2} \mathrm{D}_{3}$.

Results ROI production from preterm and term neonatal neutrophils incubated with LPS alone was not significantly increased in contrast to adults. However pre-incubation with 1,25 $(\mathrm{OH})_{2} \mathrm{D}_{3}$ before adding LPS demonstrated a significant increase $(\mathrm{p}=0.001)$ in ROI production for both preterm and term infants while simultaneous LPS and 1,25(OH)2D3 had no effect. Conclusion New born infants were hypo-responsive in the presence of sepsis in vitro which recovered on pre-treatment with 1 ,
$25(\mathrm{OH})_{2} \mathrm{D}_{3}$. Pre-treatment with vitamin $\mathrm{D}$ may improve term and preterm infants' antibacterial responses.

\section{PS-304 NEONATAL ENTEROVIRUS INFECTIONS REPORTED IN FRANCE, 2012}

${ }^{1} \mathrm{~V}$ Biran, ${ }^{2}$ S Schuffenecker, ${ }^{3} \mathrm{~J}$ Aberchih, ${ }^{4} \mathrm{~L}$ Josset, ${ }^{4} \mathrm{~L}$ Bruno, ${ }^{5} \mathrm{O}$ Baud, ${ }^{5} \mathrm{~S}$ Soudée. ${ }^{1}$ Neonatal Intensive Care Unit, Robert Debré Hospital, Paris, France; ${ }^{2}$ Centre National de Référence Des Enterovirus Et Parechovirus Laboratoire de Virologie, Centre de Biologie Est, Bron, France; ${ }^{3}$ Neonatal Intensive Care Unit, Hopital Robert Debré, Paris, France; ${ }^{4}$ Centre National de Référence Des Enterovirus Et Parechovirus Laboratoire de Virologie, Centre de Biologie Est, Bron, France; ${ }^{5}$ Neonatal Intensive Care Unit, Hôpital Robert Debré, Paris, France

\subsection{6/archdischild-2014-307384.603}

Enteroviruses (EV) are among the most common viruses infecting humans. One third of EV infections concern children under 1 year. Neonatal EV infections lead to a wide range of clinical manifestations, from mild febrile illness to severe, potentially fatal sepsislike conditions with multiorgan failure.

EV detections by serotype were reported by the "National Reference Centre for Infections EV" Lyon, on a monthly basis. Demographic, clinical and biological data are also collected in neonates hospitalised in 2012 for EV infection. Two sub-groups were identified according to the beginning of symptoms before or after 8 days of life (D8). There were 120 neonatal EV infections. Before D8, children with severe infection were born more prematurely with a low birth weight. EV most commonly detected in neonates included CVB4 and E11. Risk factors of severe EV infections included liver (73\% before D8) and haematological damages (thrombocytopenia $82 \%$ and coagulopathy $64 \%$ before D8).

This study suggest a systematic serotyping of neonatal EV infections and biological monitoring of liver function to early identification of children at high risk of clinical severity and fatality.

\section{PS-305 HUMAN PARECHOVIRUS 3 AS A CAUSE OF NEONATAL INFECTION}

${ }^{1}$ A Cilla, ${ }^{2}$ J Arnaez, ${ }^{2}$ J Suarez, ${ }^{3} \mathrm{G}$ Megias, ${ }^{1} \mathrm{P}$ Alamillo, ${ }^{4} \mathrm{M}$ Cabrerizo. ${ }^{1}$ Pediatrics, Hospital Universitario de Burgos, Burgos, Spain; ${ }^{2}$ Neonatology Pediatrics, Hospital Universitario de Burgos, Burgos, Spain; ${ }^{3}$ Microbiology, Hospital Universitario de Burgos, Burgos, Spain; ${ }^{4}$ Microbiology, Centro Nacional de Microbiología Instituto de Salud Carlos III, Madrid, Spain

\subsection{6/archdischild-2014-307384.604}

Background Human parechoviruses (HPeVs) belong to Parechovirus genus and have recently been added to the Picornaviridae family. Their epidemiology, pathogenicity and virulence is only beginning to be understood. Neonates, infants and young children seem to be the most susceptible subjects. The clinical presentation is similar to that of enterovirus infections. HPeV type 3 has been reported to cause neonatal infection, presenting with central nervous system symptoms or a sepsis-like illness.

Objective As part of a prospective study on neonatal sepsis, we aimed to assess the importance of $\mathrm{HPeV}$ as a cause of infection in the neonatal period.

Materials and methods During the period October 2012 December 2014, term newborns (0-28 days) admitted to the 
NICU for a suspected infection (defined by clinical or analytical sepsis, fever without a source, or neurological symptoms) are included in the study. Neonates with clear respiratory or gastrointestinal symptoms are excluded. A screening for bacterial and viral infection is performed. In negative cases, a real-time-RT-PCR for HPeV detection is performed in cerebrospinal fluids.

Results We present the data for the first 18 months of the study period. 28 newborns were included in the study. There were 2 confirmed cases of HPeV-3 infection. Both presented in May 2013, with fever of unknown origin, and had normal cerebrospinal fluid analysis. Case 1 was 6 days old. Case 2 was 19 days old and during the hospitalisation period developed diarrhoea and respiratory apneas requiring oxygen support. Both had a favourable evolution.

Conclusions Clinicians should include $\mathrm{HPeV}$ in the differential diagnosis of neonatal infection.

\section{PS-306 NEONATAL LUMBAR PUNCTURE - ARE CLINICAL LANDMARKS ACCURATE?}

${ }^{1}$ BJW Baxter, ${ }^{1} \mathrm{~J}$ Evans, ${ }^{1} \mathrm{R}$ Morris, ${ }^{1} \mathrm{U}$ Ghafoor, ${ }^{1} \mathrm{M}$ Nana, ${ }^{1} \mathrm{~T}$ Weldon, ${ }^{2} \mathrm{G}$ Tudor, ${ }^{1} T$ Hildebrandt. ${ }^{1}$ Paediatrics and Neonatology, Princess of Wales Hospital, Bridgend, UK; ${ }^{2}$ Paediatric Radiology, Princess of Wales Hospital, Bridgend, UK

\subsection{6/archdischild-2014-307384.605}

Background The intercristal line, defined by the superior aspect of the iliac crest, is used to clinically identify the correct position for lumbar puncture in neonates. Accepted practice is to insert the needle in the intervertebral space at L3/4 or L4/5.

Aim We hypothesised variation in anatomical structures in term neonates and that the intercristal line might not accurately aid identification of the correct intervertebral space.

Method Following ethical approval 30 term neonates were recruited. Paediatricians identified and marked the intercristal line and intervertebral space above, with neonates in left lateral position. The anatomical position of both points and the conus medullaris end point were confirmed using ultrasonography.

Results The intercristal line was marked from L2/3 to L5/S1. In 25 neonates $(83 \%)$ the intercristal line was identified between $\mathrm{L} 3 / 4$ and $\mathrm{L} 4 / 5$. The intervertebral space above this line was marked between L1/2 to L4/5. The potential site for lumbar puncture was identified too high in 11 cases (36\%).

The end of the conus medullaris ranged from L1 to L3 terminating at L2 or lower in 11 cases (36\%).

Conclusion There is wide variation in the position of the intercristal line and potential lumbar puncture site. The potential lumbar puncture site was marked higher than anticipated at L2/3 or above in 11 neonates (36\%). The end of the conus medullaris was identified in 11 neonates (36\%) at L2 or lower. Using the intercristal line to guide lumbar puncture does not appear to be accurate raising the possibility of potential spinal cord damage.

\section{PS-307 NATIONAL RECOMMENDATIONS FOR THE INVESTIGATION OF EARLY ONSET NEONATAL SEPSIS: LUMBAR PUNCTURE INCIDENCE AND INDICATIONS}

R Irving, P Lister, D Jolley, LF Hamilton, HE Gillet, JW Davis. Neonatology, St Michael's Hospital University Hospitals Bristol, Bristol, UK

\subsection{6/archdischild-2014-307384.606}

Background Early onset sepsis (EOS) is a leading cause of morbidity and mortality in newborns. The incidence of culture- positive EOS in UK neonates is 0.9/1000 live births. We aimed to compare EOS investigation practice with The National Institute of Clinical Excellence (NICE) guidelines (2012) for investigation, and determine factors that influence LP acquisition.

Method We conducted a retrospective observational study in a regional neonatal unit, using data from 213 babies investigated for EOS $(<48 \mathrm{~h})$. We reviewed patient notes, electronic records, and pathology results in 2 epochs (September-October 2012, April-May 2013). Clinical concern of infection was judged retrospectively using NICE criteria (any 'Red Flag', $>2$ risk factors, or other clinical indicators).

Results Data was collected from 213 term babies (228 infection episodes). Clinical concern for infection was identified in 52/ 228. These infants had more respiratory distress and shock than those without clinical concern. $(p<0.05)$. In total 31/228 babies had an LP; 14/31 infants had clinical concern of infection. Clinical concern for infection was similar in the 'LP group' and 'nonLP group'. The 'LP group' had a larger proportion of maternal pyrexia $(18 / 31$ vs. $60 / 137, \mathrm{p}<0.05)$ and median maximum CRP (19 mg/l vs. $1 \mathrm{mg} / \mathrm{l}, \mathrm{p}<0.05)$ than the 'non-LP group'. There were no positive blood/CSF cultures.

Conclusion Despite its low incidence EOS causes substantial clinical concern. LPs were not carried out in a number of infants despite meeting NICE criteria. Maternal risk factors and maximum CRP appear to determine the acquisition of LP more than physician 'concern' of infection.

\section{PS-308 NEONATAL BACTERIAL MENINGITIS IN A DEVELOPING COUNTRY}

F Kamoun, S Loukil, L Sfaihi, S Ben Ameur, TH Kamoun, M Hachicha. Pediatrics Department, Hedi Chaker Hospital, Sfax, Tunisia

10.1136/archdischild-2014-307384.607

Background and aims Neonatal bacterial meningitis (NBM) is a serious disease with high morbidity and mortality rates.

Aims Study the epidemiological, clinical, bacteriological aspects and the outcome of NBM.

Methods We report a retrospective analysis of 55 cases of NBM hospitalised in the paediatric department between 1990 and 2012. Inclusion criteria were infants less than 29 days of age who were hospitalised for bacterial meningitis diagnosed on either the presence of bacteria in cerebrospinal fluid (CSF) or with more than 30 cells $/ \mathrm{mm}^{3}$, predominance of neutrophils, the protein level greater than $1.2 \mathrm{~g} / \mathrm{l}$ and hypoglycorachia.

Results The mean age of diagnosis was 11 days and the sex ratio was 1.75 . The patients were premature in $9 \%$ and low birth weight in $20 \%$ of cases. The main circumstances of discovery were fever (69.1\%), refusal to breastfeed (49.1\%) and seizures (16.4\%). A myelomeninguolcele was present in 6 patients. Blood culture was positive in $34.8 \%$ of cases and the CSF culture was positive in $54.4 \%$ of cases. The main bacteria isolated was Escherichia coli (7 cases), Streptococcus B (7 cases) and Pseudomonas aeruginosa (5 cases). The cefotaximeampicillingentamicin combination was the most prescribed first-line. Ofloxacin was associated initial antibiotic therapy in 9 cases. The mortality rate was $40 \%$ and the sequelae rate in survivors was $27 \%$.

Conclusion This study emphasises the severity of NBM with high rates of mortality and neurological sequelae. An early diagnosis and effective antibiotic therapy is needed to improve the prognosis. 